\title{
Persistent Positional Vertigo in a Patient with Sudden Sensorineural Hearing Loss: A Case Report
}

\author{
Yong Won Kim, Jung Eun Shin, Yong-Sik Lee, and Chang-Hee Kim \\ Department of Otorhinolaryngology-Head and Neck Surgery, Konkuk University Medical Center, \\ Konkuk University School of Medicine, Seoul, Korea
}

$\begin{array}{ll}\text { Received } & \text { March 25, 2015 } \\ \text { Revised } & \text { May 1, 2015 } \\ \text { Accepted } & \text { May 23, 2015 }\end{array}$

Address for correspondence

Chang-Hee Kim, MD, PhD

Department of Otorhinolaryngology-

Head and Neck Surgery,

Konkuk University Medical Center,

Konkuk University

School of Medicine,

120-1 Neungdong-ro, Gwangjin-gu,

Seoul 05030, Korea

Tel $+82-2-2030-7666$

Fax +82-2-2030-5299

E-mail20110552@kuh.ac.kr
Because inner ear organs are interconnected through the endolymph and surrounding endolymphatic membrane, the patients with sudden sensorineural hearing loss (SSNHL) often complain of vertigo. In this study, we report a patient with SSNHL accompanied by persistent positional vertigo, and serial findings of head-roll tests are described. At acute stage, headroll test showed persistent geotropic direction-changing positional nystagmus (DCPN), which led to a diagnosis of SSNHL and ipsilateral light cupula. Although vertigo symptom gradually improved, positional vertigo lasted for more than 3 weeks. At this chronic stage, persistent apogeotropic DCPN was observed in a head roll test, which led to a diagnosis of the heavy cupula. Although the mechanism for the conversion of nystagmus direction from geotropic to apogeotropic persistent DCPN is unclear, the change of specific gravity of the endolymph might be one of the plausible hypothetical explanations. J Audiol Otol 2015;19(2):104-107

KEY WORDS: Sudden sensorineural hearing loss · Direction-changing positional nystagmus · Light cupula $\cdot$ Heavy cupula $\cdot$ Head-roll test $\cdot$ Positional vertigo.

\section{Introduction}

Although the etiology of sudden sensorineural hearing loss (SSNHL) is still unclear, viral neurolabyrinthitis has been accepted as one of the most common causes. Because inner ear end organs are interconnected through endolymph and surrounding membranes, both cochlear and vestibular functions can be impaired simultaneously by neurolabyrinthitis. Up to $60 \%$ of SSNHL patients may have accompanying vertigo [1]. When positional vertigo is associated with SSNHL, SSNHL accompanying ipsilateral posterior semicircular canal benign paroxysmal positional vertigo (BPPV) or persistent geotropic direction-changing positional nystagmus (DCPN) on head-roll test has been reported $[2,3]$.

In the present study, we report a patient with SSNHL with persistent positional vertigo who showed transition from geotropic to apogeotropic persistent DCPN in supine head-

This is an Open Access article distributed under the terms of the Creative Commons Attribution Non-Commercial License (http://creativecommons. org/licenses/by-nc/3.0/) which permits unrestricted non-commercial use, distribution, and reproduction in any medium, provided the original work is properly cited. roll test. Characteristic positional nystagmus findings and clinical features of the patients will be described, and possible mechanisms explaining conversion of nystagmus direction will be addressed.

\section{Case Report}

A 57-year-old woman developed sudden deafness and tinnitus in her left ear, which was followed by severe positional vertigo on the same day. Vertigo was aggravated by change of head position. The tympanic membrane was normal. Spontaneous nystagmus was not observed, and head impulse test showed no catch-up saccade. Neurological examination revealed no focal neurological deficit, and brain MRI showed no abnormal finding (Fig. 1). Pure tone audiometry (PTA) revealed an average threshold (at 500, 1000, 2000, and $3000 \mathrm{~Hz}$ ) of $99 \mathrm{~dB}$ on the left side (Fig. 2A). The patient complained of vertigo that was aggravated by turning the head to either side from a supine position. When the patient's head was turned $90^{\circ}$ to the right (Fig. 3A) or left (Fig. 3B) in a supine position, persistent geotropic DCPN was observed. The intensity of nystagmus was stronger on the left (maximal slow-phase 
velocity $=21 \%$ ) than the right (maximal slow-phase velocity $=6 \%$ s). Weak right-beating nystagmus was observed in the supine position, and the null plane, at which nystagmus stops [4-8], was identified on the left side, which led to a diagnosis of light cupula with ipsilateral SSNHL [3]. At each position, we observed positional nystagmus for at least 2 minutes. A caloric test revealed no canal paresis. The patient was administered systemic steroids and vestibular suppressants. During 7 days of hospitalization, positional vertigo was gradually improved, and the intensity of persistent geotropic DCPN also gradually diminished.

Two weeks after discharge from the hospital (3 weeks after symptom onset), the patient returned and reported that left hearing was much improved, but mild positional vertigo remained. PTA showed an average threshold of $30 \mathrm{~dB}$ on the

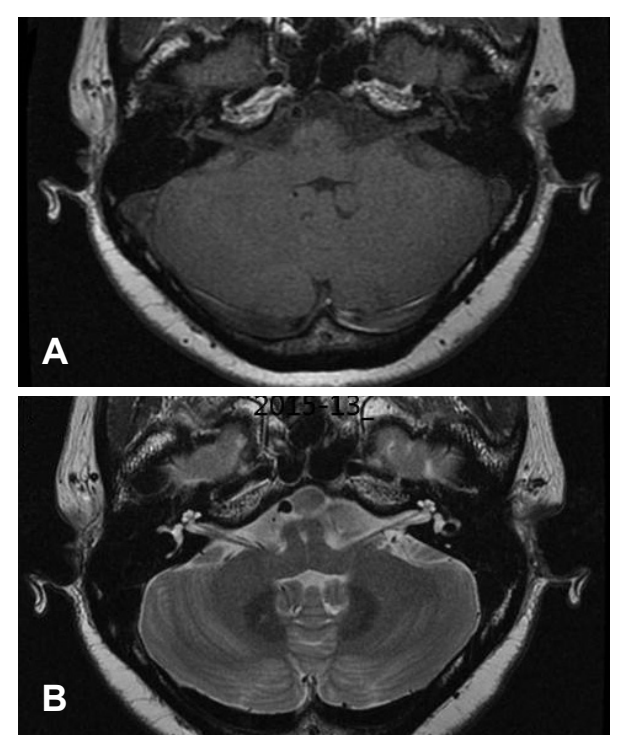

Fig. 1. Magnetic resonance imaging revealed no abnormal findings within the inner ear and cerebellum in T1 (A) and T2-weighted (B) axial images. left ear (Fig. 2B). Head-roll test revealed persistent apogeotropic DCPN (Fig. 3C, D). A canalith repositioning procedure (CRP) was performed considering the diagnosis of cupulolithiasis of the left horizontal semicircular canal (hSCC). Despite repeated CRP sessions, persistent apogeotropic DCPN on head-roll test continued, and lasted for 10 days thereafter.

\section{Discussion}

Viral infection, labyrinthine ischemia, labyrinthine hemorrhage, and disruption of the cochlear membrane have been suggested as possible causes of SSNHL [9]. Considering that the lesion site lies within the inner ear organs [9], dysfunction of both the cochlea and vestibules may concurrently occur in SSNHL. DCPN during the head-roll test is typically observed in hSCC BPPV. DCPN with a prolonged duration is highly indicative of the presence of cupulopathy (either a light or heavy cupula). A light cupula, which is a cupula with a lower specific gravity than the surrounding endolymph, was recently introduced to explain persistent geotropic DCPN [4,6$8,10,11]$. When persistent apogeotropic DCPN is observed in a head-roll test, otolith debris attached to the hSCC cupula (cupulolithiasis) has been accepted as an underlying mechanism. In cases with both persistent geotropic and apogeotropic DCPN, the affected side can be determined by identifying the side of the null plane $[12,13]$.

Recently, it was reported that the condition of light cupula may be responsible for the positional vertigo which is accompanied by ipsilateral SSNHL [3,14]. The pathophysiology of light cupula still remains unclear. The attachment of light debris, which has not been identified yet, onto the cupula has been suggested as a cause of the light cupula [7], which was further supported by recent findings that the angle of the null plane was significantly greater in the light cupula than the heavy cupula assumably due to attachment of light debris onto
Fig. 2. Pure tone audiometry revealed an average threshold (at $500,1000,2000$, and $3000 \mathrm{~Hz}$ ) of $99 \mathrm{~dB}$ at the second day of symptom onset (A), and $30 \mathrm{~dB}$ at three weeks after symptom onset (B) on the left ear.

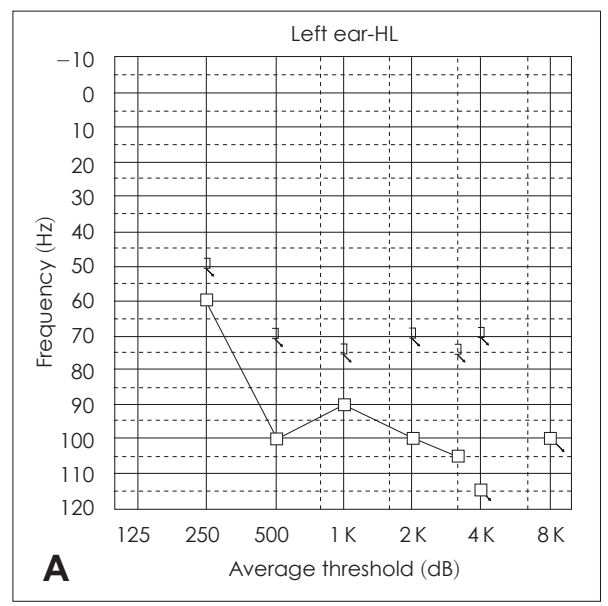

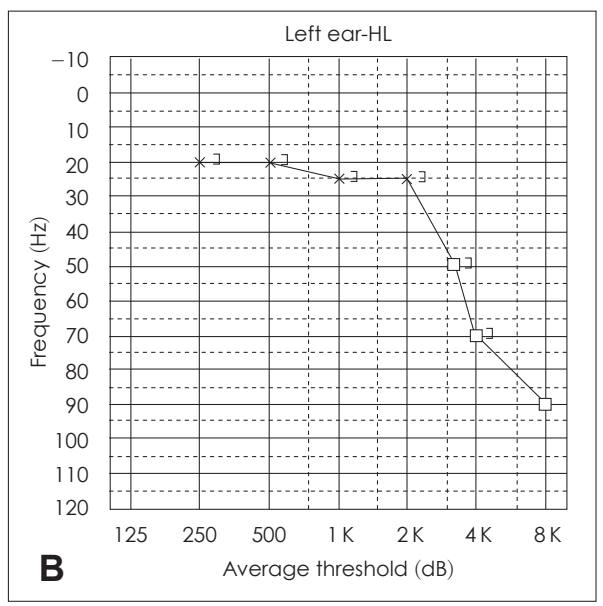




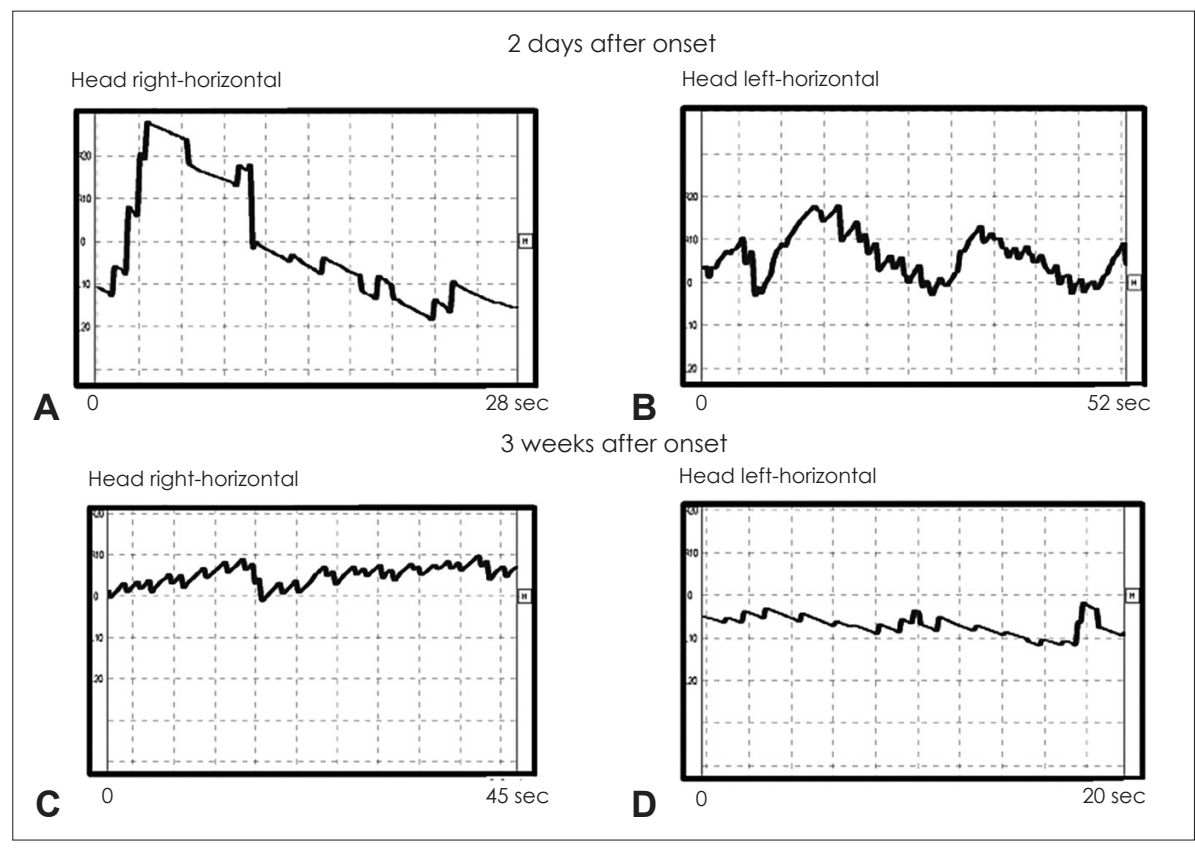

Fig. 3. Video-oculography of horizontal eye movement during a supine head-roll test. On the second day after symptom onset, head-roll test revealed persistent geotropic direction-changing positional nystagmus (DCPN). Persistent rightbeating nystagmus was observed when head-turning to the right $(A)$, and persistent left-beating nystagmus was observed when the head was turned to the left (B). At 3 weeks after symptom onset, persistent apogeotropic DCPN was observed on head-roll test $(C, D)$. the lateral side of the hSCC cupula in case of the light cupula [11]. An increase in the specific gravity of the endolymph was proposed as another mechanism of the light cupula by others [4-6], which was further supported by recent findings that a light cupula can be accompanied by SSNHL [3], and that vertical and torsional components of positional nystagmus can be observed possibly due to the involvement of light cupula phenomenon in all three semicircular canals on the same side [15]. It was speculated that increased protein content within the endolymph caused by an insult to the inner ear in patients with SSNHL may elevate the specific gravity of the endolymph, which consequently results in the condition of a light cupula [3]. A heavy cupula, in which specific gravity of the surrounding endolymph is lower than the cupula, may be a part of the pathophysiology explaining persistent apogeotropic DCPN.

Herein, the patient first presented persistent geotropic DCPN, which later changed to persistent apogeotropic DCPN. Two mechanisms may explain this condition: 1) a light cupula associated with ipsilateral SSNHL may be caused by an increase in endolymphatic specific gravity, and overcompensation of endolymphatic fluid homeostasis may cause the endolymph to have a lower specific gravity than that of the cupula, resulting in the condition of a heavy cupula; and 2) a light cupula may be caused by the attachment of light debris onto the cupula, and then the increase in density of attached light debris by any chance caused the specific gravity of the cupula to become greater, thus resulting in the condition of a heavy cupula. Considering that positional vertigo with per- sistent apogeotropic DCPN was unresolved despite repeated sessions of CRP and lasted for more than 3 weeks, we speculate that the first mechanism is more probable to explain the conversion from geotropic to apogeotropic persistent DCPN. Compared with the light cupula without SSNHL which mostly resolved spontaneous within 1-2 weeks [4], the patient in this study exhibited longer duration of positional nystagmus, which may be caused by more severe disruption of endolymphatic homeostasis in SSNHL.

In conclusion, a patient with SSNHL with vertigo suffered from persistent positional vertigo which may last for several weeks. In this patient, head-roll test revealed the transition of nystagmus direction from geotropic to apogeotropic persistent DCPN. Although the mechanism for this observation is unclear, we speculate that the change of specific gravity of the endolymph may provide more plausible hypothetical explanation.

\section{Acknowledgments}

This study was supported by Basic Science Research Program through the National Research Foundation of Korea (NRF) funded by the Ministry of Education, Science and Technology (2012R1A1A 2044883).

\section{REFERENCES}

1) Park HM, Jung SW, Rhee CK. Vestibular diagnosis as prognostic indicator in sudden hearing loss with vertigo. Acta Otolaryngol Suppl 2001;545:80-3.

2) Kim CH, Shin JE, Park HJ, Koo JW, Lee JH. Concurrent posterior semicircular canal benign paroxysmal positional vertigo in patients with ipsilateral sudden sensorineural hearing loss: is it caused by 
otolith particles? Med Hypotheses 2014;82:424-7.

3) Kim CH, Choi JM, Jung HV, Park HJ, Shin JE. Sudden sensorineural hearing loss with simultaneous positional vertigo showing persistent geotropic direction-changing positional nystagmus. Otol Neurotol 2014;35:1626-32.

4) Kim CH, Kim MB, Ban JH. Persistent geotropic direction-changing positional nystagmus with a null plane: the light cupula. Laryngoscope 2014;124:E15-9.

5) Hiruma K, Numata T, Mitsuhashi T, Tomemori T, Watanabe R, Okamoto $\mathrm{Y}$. Two types of direction-changing positional nystagmus with neutral points. Auris Nasus Larynx 2011;38:46-51.

6) Hiruma $K$, Numata $T$. Positional nystagmus showing neutral points. ORL J Otorhinolaryngol Relat Spec 2004;66:46-50.

7) Ichijo H. Persistent direction-changing geotropic positional nystagmus. Eur Arch Otorhinolaryngol 2012;269:747-51.

8) Bergenius J, Tomanovic T. Persistent geotropic nystagmus--a different kind of cupular pathology and its localizing signs. Acta Otolaryngol 2006;126:698-704.

9) Schuknecht HF, Donovan ED. The pathology of idiopathic sudden sensorineural hearing loss. Arch Otorhinolaryngol 1986;243:1-15.

10) Imai T, Matsuda $K$, Takeda N, Uno A, Kitahara T, Horii A, et al. Light cupula: the pathophysiological basis of persistent geotropic positional nystagmus. BMJ Open 2015;5:e06607.

11) Ichijo $\mathrm{H}$. Neutral position of persistent direction-changing positional nystagmus. Eur Arch Otorhinolaryngol 2015 Jan 23 [Epub]. http://dx. doi.org/10.1007/s00405-014-3487-3.

12) Kim $\mathrm{CH}$, Shin JE, Kim YW. A new method for evaluating lateral semicircular canal cupulopathy. Laryngoscope 2015 Jan 30 [Epub]. http://dx.doi.org/10.1002/lary.25181.

13) Bisdorff AR, Debatisse D. Localizing signs in positional vertigo due to lateral canal cupulolithiasis. Neurology 2001;57:1085-8.

14) Seo T, Saito K, Doi K. Intractable persistent direction-changing geotropic nystagmus improved by lateral semicircular canal plugging. Case Rep Otolaryngol 2015;2015:192764.

15) Kim CH, Shin JE, Shin DH, Kim YW, Ban JH. "Light cupula" involving all three semicircular canals: a frequently misdiagnosed disorder. Med Hypotheses 2014;83:541-4. 